\title{
Deficiencia congénita del factor VII de la coagulación: relato de casos de una institución de atención ambulatoria
}

\section{Congenital deficiency of coagulation factor VIl: report of cases of an ambulatorial attendance institution}

\author{
Thaís Byanca Brandenburg,' Reginaldo Jose Andrade, ${ }^{2}$ \\ Antônio Carlos Michels De Oliveira, ${ }^{3}$ Francisco Schossler Losss
}

\begin{abstract}
Brandenburg TB, Andrade RJ, ACM De Oliveira, Loss FS. Deficiencia congénita del factor VII de la coagulación: relato de casos de una institución de atención ambulatoria. Rev Soc Peru Med Interna. 2019;32(2):54-58. https://doi.org//0.36393/spmi.v32i2.218
\end{abstract}

\begin{abstract}
RESUMEN
INTRODUCCIón. La deficiencia del factor VII (FVII) de la coagulación es una coagulopatía hereditaria rara que presenta amplia diversidad genética y manifestaciones hemorrágicas de grado variable, desde situaciones asintomáticas hasta episodios hemorrágicos potencialmente letales. El diagnóstico se realiza por exámenes de laboratorio de una muestra de sangre con pruebas de rutina y dosificación del factor. El tratamiento se determina de acuerdo con la correlación entre la dosificación del factor y las manifestaciones hemorrágicas de la enfermedad. OвJeтIvo. El estudio abordó la revisión de los casos de deficiencia del FVII, abarcó aspectos clínicos, diagnósticos y terapéuticos de la enfermedad. Material y Métodos. Casos de pacientes que fueron reportados con deficiencia congénita del FVII en un ambulatorio. El estudio fue realizado con una revisión de los prontuarios de seis pacientes, registrados en el Hemepar en Cascavel/Paraná. Se realizó un análisis de la literatura basada en artículos de la base de datos PubMed y Scielo, así como el Manual de Coagulopatías Raras del Protocolo del Ministerio de Salud Brasileña. Investigación sometida y aprobada por el Comité de Ética en Investigación con Seres Humanos de la Fundación Asís Gurgacz portando CAAE 90617418.3.0000.5219. Resultados. De los seis pacientes, cinco pacientes presentaron FVII mayor de $20 \%$ y uno, FVII menor de $1 \%$; cuatro no tenían antecedentes de sangrado previo, tres no tenían historia familiar de la enfermedad y cinco recibieron reposición de FVII. Conclusión. Este estudio mostró la importancia de realizar una investigación completa del paciente e individualizó cada caso para evaluar la real necesidad de reposición del FVII y evitar su desperdicio.
\end{abstract}

Palabras clave. Deficiencia, factor VII, Sangrado, Factor VII activado recombinante.

\begin{abstract}
InTRODUction. Coagulation factor VII (FVII) deficiency is a rare hereditary coagulopathy presenting with a wide genetic diversity and hemorrhagic manifestations of varying degrees, ranging from asymptomatic to even life-threatening hemorrhagic episodes. The diagnosis is made with routine blood lab tests and dosage of the factor. The treatment is determined according to the correlation between the
\end{abstract}

I. Académica del curso de Medicina, Centro Universitario Assis Gurgacz, Cascavel / PR, Brasil.

2. Médico hematólogo. Hemocentro Regional de Cascavel./ PR. Graduación en Medicina y especialización en Hematología por la Universidad Federal de Paraná, UFPR, Brasil.

3. Farmacéutico bioquímico. Hemocentro Regional de Cascavel / PR, Brasil.

4. Académico del curso de Medicina. Centro Universitário Assis Gurgacz, Cascavel/PR, Brasil dosage of the factor and the hemorrhagic manifestations. OвлестIVE.This study addresses a review of FVII deficiency cases encompassing clinical, diagnostic and therapeutic aspects of the disease. Methodology. Cases of patients were reported with congenital FVII deficiency at an outpatient clinic. The study was carried out with a review of the medical records of six patients, registered in Hemepar of Cascavel I PR. An analysis was performed of the literature based on articles of the PubMed database and Scielo, as well as the Rare Coagulopathies Manual of Protocol of the Ministry of Health. Results. From 6 patients, five patients had FVII> 20\% and only one had FVII $<1 \%$, four had no history of previous bleeding, three had no family history of the disease and five received FVII replacement. Conclusion. This study showed the importance of performing a complete investigation of the patient and individualized each case in order to evaluate the real need for FVII replacement, preventing unnecessary use.

KeYwords. Factor VII deficiency, Bleeding, Recombinant factor VIla. 


\section{INTRODUCCIÓN}

La deficiencia del factor VII (FVII) de la coagulación es una condición hereditaria rara, con una prevalencia estimada en $1: 500$ 000, sin ninguna predilección racial y de sexo.

La enfermedad es transmitida por un mecanismo autosómico recesivo con amplia diversidad genética, que resulta en un trastorno hemorrágico de gravedad variable. ${ }^{1,2}$ Más de 100 mutaciones en el gen codificador del FVII han sido descritos como responsables de la gran variación en la transcripción génica y la traducción. Esto explica la falta de correlación entre la actividad del FVII y los síntomas hemorrágicos: algunos pacientes con niveles menores de $1 \%$ pueden presentar poca 0 ninguna manifestación hemorrágica..$^{1,5,6}$ Los pacientes sintomáticos pueden desarrollar sangrados graves en los sistemas nervioso y gastrointestinal, con gran riesgo de muerte. ${ }^{5}$

Una correlación clínica y de laboratorio débil es un obstáculo para el diagnóstico, dificulta la previsión del riesgo de sangrado y el manejo de un portador de deficiencia de FVII.

La ausencia de correlación entre la actividad del FVII, la gravedad de la hemorragia y los grandes costos involucrados en el manejo profiláctico y terapéutico del disturbio hemorrágico justifican describir esa patología y presentar la clínica, laboratorio y terapéutica características de los casos revisados.

\section{MATERIAL Y MÉTODOS}

Es un estudio descriptivo, retrospectivo de una serie de casos.

Los registros médicos de seis pacientes con deficiencia congénita de FVII atendidos en el ambulatorio del Hemepar en Cascavel-Paraná, Brasil, fueron revisados. Otros dos pacientes, admitidos en la institución, fueron excluidos del análisis, debido a la falta de las mediciones de FVII en las historias clínicas.

Además, se realizó una comparación entre los pacientes presentados con deficiencia congénita de FVII y los casos reportados en la literatura, sobre los artículos incluidos en las bases de datos Pubmed y Scielo. El estudio fue aprobado por el Comité de Ética en Investigación con Seres Humanos de la Fundación Asís Gurgacz (aprobación CAAE 90617418.3.0000.5219).

\section{RESULTADOS}

Los 6 pacientes analizados fueron atendidos entre noviembre de 2008 y enero de 2018. La edad varió de 9 a 53 años, 3 pacientes fueron del sexo femenino y 3 , del sexo masculino. Entre estos pacientes, los motivos de la investigación fueron resultados preoperatorios anormales (tres pacientes), sangrado previo considerado anormal (dos pacientes) y sangrado familiar (un paciente).

La Tabla 1 enumera los pacientes analizados, clasificados por motivo de la investigación.

La primera paciente, de 26 años, fue admitida al ambulatorio en 2014. Estaba embarazada (primera gestación, 24 semanas) y refirió la identificación de la prolongación del tiempo de protrombina (TP) en la evaluación preoperatoria de una cirugía plástica (inserción de prótesis mamaria) en 2010. Refirió de que se sometió a un procedimiento odontológico, con la extracción de cuatro dientes molares, en 2005, sin sangrado excesivo, y negó cualquier antecedente familiar de sangrado anormal. La actividad del FVII fue estudiada y reportada en $38,6 \%$. Se embarazó en 2014 y para el parto fue sometida a cesárea, con reposición de FVII activado recombinante (FVIIa-r) (NovoSeven ${ }^{\circledR}$ RT, Novo Nordisk), en el preoperatorio inmediato y hasta tres días del posoperatorio.

El segundo paciente, un niño de 9 años, fue derivado después de una prolongación anormal del TP, evaluado como rutina preoperatoria para la corrección de una fisura labial. No hubo antecedentes personales o familiares de sangrado anormal. El nivel de FVII fue de 45\%. La cirugía fue realizada sin el uso de factor de coagulación sanguínea, sin ningún sangrado considerado anormal.

El tercer paciente, de 10 años, sin historia personal o familiar de sangrado, tuvo el diagnóstico de deficiencia de FVII tras estudio de coagulación para cirugía otorrinolaringológica programada (adenoidectomía y amigdalectomía). Los niveles del FVII fueron estimados en $44 \%$ y la paciente recibió FVIIa-r para el procedimiento.

Dos pacientes fueron encaminados con historia de sangrado anormal. Una paciente de 29 años, con 22 semanas de embarazo (segunda gestación), relató metrorragia desde la menarquia y un gran sangrado posparto en la primera gestación, pero no fue necesaria una transfusión. Ella mencionó parientes, no 


\begin{tabular}{|c|c|c|c|c|c|c|c|c|}
\hline \multirow[b]{2}{*}{ Sexo } & \multicolumn{7}{|c|}{ Clasificación de los pacientes } & \multirow[b]{2}{*}{ Tratamiento } \\
\hline & $\begin{array}{l}\text { Edad } \\
\text { (años) }\end{array}$ & $\begin{array}{l}\text { Actividad } \\
\text { del FVII }\end{array}$ & $\begin{array}{c}\text { Exámenes anormales } \\
\text { preoperatorios }\end{array}$ & $\begin{array}{c}\text { Sangrado anterior } \\
\text { anormal }\end{array}$ & $\begin{array}{c}\text { Historia de } \\
\text { diagnóstico familiar }\end{array}$ & $\begin{array}{l}\text { Sangrado } \\
\text { anterior }\end{array}$ & $\begin{array}{c}\text { Historia de } \\
\text { sangrado familiar }\end{array}$ & \\
\hline - Femenino & 26 & $38,6 \%$ & Sí & & & No & No & FVlla-r \\
\hline - Masculino & 9 & $45,0 \%$ & Sí & & & No & No & No realizado \\
\hline - Femenino & 10 & $44,0 \%$ & Sí & & & No & No & FVlla-r \\
\hline - Femenino & 29 & $22,0 \%$ & Sí & & Sí & Sí & FVlla-r & \\
\hline - Masculino & 53 & $<1,0 \%$ & & Sí & FVlla-r & Sí & Sí & Ácido tranexámico \\
\hline - Masculino & 22 & 47,2 & & Sí & No & Sí & CCP y FVII & \\
\hline
\end{tabular}

Factor VIl activado recombinante (FVlla-r). CCP: concentrado de complejo de protrombina

identificados y sin detalles, con historial de sangrado anormal. El TP se registró como un $32 \%$ de actividad y la actividad del FVII se determinó en un $22 \%$. Ella recibió FVIIa-r en el parto, sin sangrado posparto anormal.

El otro, paciente del sexo masculino, 53 años de edad, fue encaminado con historia de epistaxis y sangrado gingival desde el nacimiento. Él mencionó sangrado nasal frecuente en parientes (padre y hermana que por sangrado vaginal). El paciente fue admitido en el hospital después de un trauma de perforación en el pie izquierdo, con sangrado excesivo. El TP fue considerado incoagulable y la concentración de FVII fue inferior a $1 \%$. Él recibió FVIIa-r y ácido tranexámico y la evolución postoperatoria fue satisfactoria, sin sangrado anormal.

El último paciente, de 22 años de edad, del sexo masculino, fue encaminado después de que la madre fue diagnosticada con deficiencia del FVII. El paciente negó cualquier sangrado previo y procedimientos invasivos. El TP fue prolongado (datos no registrados) y la actividad del FVII fue determinada en el 47,2\%. Se le aconsejó recibir un complejo de protrombina o FVIIa-r en eventos de sangrado o procedimientos quirúrgicos.

\section{DISCUSIÓN}

El FVII es un factor de la coagulación dependiente de la vitamina $\mathrm{K}$ y responsable de la iniciación de la coagulación sanguínea. Es sintetizado en los hepatocitos y circula en el torrente sanguíneo de dos formas, una inactiva o zimógeno (99\%) y una pequeña fracción (1\%) activada o enzimática. ${ }^{1}$ Esta última es responsable del inicio de la coagulación in vivo. ${ }^{3}$ El inicio de la hemostasia secundaria en un sitio de lesión endotelial ocurre cuando el factor tisular (FT), normalmente presente en el subendotelio, se expone a la sangre después de una lesión con desnudamiento del endotelio. El FT interactúa con el FVII activado (FVIIa) de la corriente sanguínea y forma el complejo FT-FVIIa, en presencia de calcio. Este complejo, también conocido como vía extrínseca, activa cantidades mínimas de los factores IX y X presentes en el lugar de la lesión. ${ }^{3} \mathrm{El}$ factor X activado (FXa) se une al factor $\mathrm{V}$ (cofactor) $\mathrm{y}$ en la presencia de calcio forman el complejo protrombinasa, capaz de convertir la protrombina (factor II) en trombina. ${ }^{3}$ La cantidad de trombina generada es esencial para la amplificación del proceso de coagulación. El complejo vía intrínseca (FIXa ligado al factor VIII) activa una cantidad mayor de FX que al conectarse al FVa convierte gran cantidad de protrombina en trombina y fibrinógeno en fibrina, para formar una red estabilizada por el FXIII y, finalmente, el coágulo. ${ }^{3}$

La deficiencia cuantitativa o cualitativa del FVII de la coagulación sirve como base para la clasificación de la coagulopatía. La enfermedad del tipo I se caracteriza por la reducción de la actividad funcional (FVII:C) y el nivel antigénico (FVII:Ag) del FVII. La enfermedad del tipo II se expresa como un defecto cualitativo, que resulta en la transcripción de una proteína disfuncional: hay reducción en la actividad del FVII:C, pero el FVII:Ag detectado en la concentración es normal. ${ }^{1,5}$

La hemorragia grave ocurre en alrededor de $10 \%$ a $15 \%$ de los pacientes y otro $30 \%$ no presenta sangrado con riesgo de muerte. ${ }^{5}$ Los pacientes sintomáticos presentan sangramientos que involucran principalmente los 
sistemas nervioso central y gastrointestinal, pueden ser serios y con gran riesgo de muerte. ${ }^{5}$

El sangrado generalmente se caracteriza por equímosis, hematomas y epistaxis. La menorragia y hemorragia postparto pueden ser clínicamente significativa. ${ }^{1}$ Las hemartrosis, hemorragia retroperitoneal, hemorragia gastrointestinal e intracraneal generalmente ocurren en pacientes con niveles plasmáticos muy bajos. Algunos pacientes con niveles inferiores a $1 \%$ pueden, sorprendentemente, desarrollar hemorragias significativas, ${ }^{1,5,6}$ pero el paciente en estudio con este nivel presentó solo sangrado gingival y epistaxis.

Las pruebas de selección para evaluar una diátesis hemorrágica sospechosa incluyen, además del recuento de plaquetas y estudios de la coagulación como tiempo de protrombina (TP), tiempo de trombina (TT) y tiempo de tromboplastina parcial activado (TTPA). Entre los ensayos de rutina, los pacientes con deficiencia de FVII pueden presentar TP de actividad prolongada de la protrombina, mientras que el TTPA y el TT están dentro de los límites normales. ${ }^{1,2,5}$

La actividad normal del FVII varía de 55\% a $180 \%$. El diagnóstico de la deficiencia se hace por la identificación de la actividad reducida del FVII (FVII:C). La diferenciación entre los tipos I y II es hecha por la cuantificación antigénica del FVII (FVII:Ag), pero esto no permite prever la tendencia del sangrado.,

Está bien establecido que la correlación entre el factor de nivel de actividad y el riesgo de hemorragia es bajo por lo que es difícil de predecir el riesgo de sangrado portador de la deficiencia de FVII. ${ }^{1}$ Un control hemostático normal del sangrado generalmente ocurre con actividad de FVII por encima de $15 \%$ a $20 \%$ : individuos con actividad funcional por encima de 15 a $20 \%$ raramente sangran o necesitan reposición de factor. $^{1,2}$

Los eventos hemorrágicos clínicamente importantes generalmente ocurren solo con actividad funcional por debajo del 10 al $15 \%$, pero datos de la literatura muestran que la correlación entre los niveles de FVII niveles y el riesgo de hemorragia no es lineal. ${ }^{1} \mathrm{~A}$ pesar de las evidencias de que el sangrado aumenta en pacientes con concentración de factor menor que el $15 \%$, algunos pacientes con actividad por debajo de $1 \%$ eventualmente no presentan hemorragia. ${ }^{1,5}$

El tratamiento de elección para el reemplazo del FVII es el FVIIa-r, a una dosis de 15 a $30 \mu \mathrm{g} / \mathrm{kg}$ de peso corporal. ${ }^{1}$ Una dosis única puede ser suficiente en caso de hemorragias leves o moderadas, como en hemartrosis y extracciones dentales.

La reposición puede ser prescrita cada 6 a $8 \mathrm{~h}$ por uno a tres días después de pequeñas cirugías. Se administra a intervalos más cortos (cada 4 a 6 h) y durante un período más largo (7 a 10 días) en cirugías mayores. ${ }^{1,5,6}$

El plasma fresco congelado (FFP) en la dosis de $10 \mathrm{ml} /$ $\mathrm{kg}$ de peso corporal cada 4 a 6 horas es un tratamiento alternativo: su disponibilidad es mayor y su costo es mucho menor, pero las infusiones repetidas se asocian al riesgo de sobrecarga hídrica. El concentrado del complejo de protrombina (CCP) es otra opción que puede administrarse a la dosis de $10 \mathrm{UI} / \mathrm{kg}$ cada 4 a $6 \mathrm{~h}$, pero este componente nunca debe estar asociado al antifibrinolítico debido al gran riesgo trombogénico.,6

Los antifibrinolíticos deben ser prescritos en procedimientos odontológicos. ${ }^{1,6}$

El presente estudio relata seis pacientes, la mitad de ellos evaluados después de la identificación de un TP prolongado en estudios preoperatorios. Se notó que un paciente que fue previamente sometido a cirugía odontológica extensa sin complicaciones hemorrágicas había recibido el FVII profiláctico recombinante durante una cesárea. Se destaca el uso del FVII profiláctico en la cirugía otorrinolaringológica en paciente con actividad FVII del $44 \%$ sin evidencia de sangrado previo. Esto genera preocupación y es cuestionable la notificación a un paciente con actividad prolongada de TAP y FVII del $47 \%$ sobre la necesidad de reposición de factor en la presencia de sangrado.

La prescripción de factor suplementario en los dos primeros pacientes y la recomendación de eventual uso en el tercer paciente aparentemente no es una recomendación estándar para el manejo de pacientes con deficiencia de FVII. Como el valor del FVIIa-r es extremadamente alto, actualmente en torno de 5650 dólares cada frasco con $5 \mathrm{mg}$ (costo estimado para una infusión única de un paciente de $70 \mathrm{~kg}$ es de 1280 dólares), esa reposición debe ser de uso restringido para los pacientes que realmente lo necesiten., ${ }^{4,7}$

Los resultados del Seven Treatment Evaluation Registry (STER) mostraron que la profilaxis con FVIIa-r, tres veces por semana o una dosis semanal de $90 \mu \mathrm{g} / \mathrm{kg}$ es efectiva en pacientes con discapacidad grave. ${ }^{4}$ A largo plazo, la profilaxis primaria se indica en niños con 
discapacidad grave, y la profilaxis secundaria puede ser indicada para pacientes con hemartrosis recurrente 0 hemorragia intracraneal. ${ }^{1,5}$

En conclusión, este estudio destaca que la deficiencia congénita del FVII no implica necesariamente en aumento del riesgo de sangrado incluso en procedimientos invasivos. La falta de conocimiento sobre el diagnóstico y el tratamiento de enfermedades raras generalmente conduce al uso inadecuado de medicamentos. La posibilidad de que un paciente necesite el tratamiento con FVII activado recombinante (FVIIa-r), ((NovoSeven ${ }^{\circledR}$ RT, Novo Nordisk)), que es extremadamente caro, refuerza la necesidad de una investigación más adecuada de cada caso, incluyendo exámenes de laboratorio, registro de eventos hemorrágicos y conductas efectivas realizadas después del diagnóstico.

\section{REFERENCIAS BIBLIOGRÁFICAS}

I. Brasil. Ministério da Saúde. Manual das coagulopatias hereditárias raras. Brasília: 2015. 33-37p.

2. Brasil. Ministério da Saúde. Manual de Diagnóstico Laboratorial das Coagulopatias Hereditárias e Plaquetopatias. Brasília: 2016.86p.

3. Ferreira CN, Souza MO, Dusse LMS, Carvalho MG. O novo modelo da cascata de coagulação baseado nas superfícies celulares e suas implicações. Rev Brasil Hematol Hemoter. 2010;32(5):4I6-42I.

4. Napolitano M, Blaizot MG, Dolce A, Schved JF, Auerswald G, Ingerslev J, et al. Prophylaxis in congenital factor VII deficiency: indications, efficacy and safety. Results from the Seven Treatment Evaluation Registry (STER). Haematologica. 2013;98(4):538-544.

5. Napolitano M, Siragusa S, Mariani G. Factor VII deficiency: Clinical phenotype, genotype and therapy.J Clin Med. 20।7;6(4):38.

6. Palla R, Peyvandi F, Shapiro AD. Rare bleeding disorders: diagnosis and treatment. Blood. 20I5; I25(I3):2052-206I. URL disponible en: www. bloodjournal.org. (Acceso: I6 de março de 2018).

7. Dutton RP, Hess JR, Thomas MS. Recombinant factor VIla for control of hemorrhage: early experience in critically III trauma patients. J Clin Anaesth. 2003;15:184-88.

CORRESPONDENCIA:Thaís Byanca Brandenburg thaisbyanca@gmail.com

FECHA DE RECEPCIÓN: 23 de marzo de 2019.

FECHA DE ACEPTACIÓN: 20 de mayo de 2019.

DECLARACIÓN DE CONFLICTO DE INTERESES: ninguno, según los autores.

FINANCIAMIENTO: por los autores. 